\title{
Avaliação da Reprodutibilidade na Identificação de Pontos Anatômicos Obtidos em Telerradiografias Laterais de Crânios Secos Com e Sem Identificadores Metálicos
}

Assessment of Reproducibility in the Identification of Anatomical Landmarks Obtained from Lateral Cephalogram of Dry Skulls With and Without Metal Handles

Nádia Assein Arús ${ }^{1}$, Mariana Boessio Vizzotto ${ }^{2}$, Priscila Fernanda da Silveira ${ }^{3}$, Leticia Ruhland Correa ${ }^{4}$, Heraldo Luis Dias da Silveira ${ }^{5}$, Heloísa Emília Dias da Silveira ${ }^{6}$

\begin{abstract}
Objectives: This study evaluated cephalometric measurements from lateral chephalograms of dry skulls with and without metal points on anatomical landmarks.

Materials and methods: Ten dry skulls were used for the acquisition of twenty radiographs divided into two groups: ten radiographs with metal points on anatomical landmarks (gold standard) and ten without the identifiers. A specialist in oral radiology, trained and calibrated, carried out the cephalometric analysis in both groups. Comparisons were performed using paired t-test and Bland \& Altman coefficient of repeatability.

Results: The results of repeatability coefficient showed statistically differences in some cephalometric measurements performed in cephalograms without the metal markers.

Conclusion: An examiner, even if calibrated, is subject to errors in cephalometric measurements. Therefore, the intra-examiner calibration may not reflect certainty of accuracy but the repetition of errors.
\end{abstract}

Keywords: reproducibility, cephalometry, anatomical points, imaging diagnosis.

\section{Resumo}

Objetivo: Este estudo "in vitro" avaliou comparativamente as medidas cefalométricas realizadas em telerradiografias laterais de crânios secos sem e com identificadores metálicos nos pontos anatômicos. Materiais e métodos: Foram utilizados 10 crânios secos para a realização de 20 telerradiografias divididas em dois grupos: 10 telerradiografias com identificadores metálicos nos pontos anatômicos (padrão-ouro) e 10 sem identificadores. Um especialista em radiologia odontológica, treinado e calibrado, realizou os traçados cefalométricos nos dois grupos. As comparações das medidas foram realizadas por meio do teste t pareado e coeficiente de repetibilidade de Bland\&Altman.

Resultados: Os resultados mostraram existir diferenças estatisticamente significativas, pela avaliação do coeficiente de repetibilidade, entre os valores de algumas medidas realizadas sobre as telerradiografias com os identificadores metálicos (padrão-ouro) daquelas realizadas na ausência destes.

Conclusão: Pode-se concluir que, mesmo treinado e calibrado, o profissional pode gerar valores incorretos para os fatores cefalométricos. Assim, a calibragem intra-examinador não reflete a certeza do acerto, e sim a repetição de erros.

Palavras-chave: diagnóstico por imagem, pontos de referência anatômicos, medidas, radiografia.
${ }^{1}$ Mestra em Odontologia; Departamento de Cirurgia e Ortopedia; Faculdade de Odontologia da UFRGS, Porto Alegre, RS, Brasil.

${ }^{2}$ Doutora em Odontologia, Departamento de Cirurgia e Ortopedia; Faculdade de Odontologia da UFRGS, Porto Alegre, RS, Brasil.

${ }^{3}$ Aluna do curso de Mestrado em Odontologia; Departamento de Cirurgia e Ortopedia; Faculdade de Odontologia da UFRGS, Porto Alegre, RS, Brasil.

${ }^{4}$ Mestra em Odontologia; Departamento de Cirurgia e Ortopedia; Faculdade de Odontologia da UFRGS, Porto Alegre, RS, Brasil.

${ }^{5}$ Doutor em Odontologia, Professor Adjunto da Faculdade de Odontologia da UFRGS; Departamento de Cirurgia e Ortopedia; Faculdade de Odontologia da UFRGS, Porto Alegre, RS, Brasil.

${ }^{6}$ Doutora em Odontologia, Professora Associada da Faculdade de Odontologia da UFRGS; Departamento de Cirurgia e Ortopedia; Faculdade de Odontologia da UFRGS, Porto Alegre, RS, Brasil.

Correspondência: Priscila Fernanda da Silveira

Endereço: Faculdade de Odontologia - Universidade Federal do Rio Grande do Sul Rua Ramiro Barcelos 2492, 5o andar - Radiologia, CEP 90035-003 - Porto Alegre, RS, Brasil

Fone: (51) 33085199

E-mail: prifsilveira@yahoo.com.br

Data de Submissão: 30/04/2012

Data de Aceite: $23 / 05 / 2012$

\section{Introdução}

As telerradiografias laterais evidenciam acidentes anatômicos sobrepostos em duas dimensões e, em razão disto, há uma dificuldade na identificação dos pontos pelo profissional, podendo gerar erros nas medições angulares e lineares das análises cefalométricas (STEINER, 1953; YEN, 1960; STABRUN; DANIELSEN, 1982; RUDOLPH; SINCLAIR; COGGINS, 1998). Estudos avaliaram a influência do examinador na reprodutibilidade e nos erros, destacando a grande variabilidade nas avaliações interexaminadores, salientado ainda a importância da calibração destes profissionais (MAJOR et al.,1994; LAU; COOKE; HÄGG, 1997; ALBUQUERQUE JÜNIOR; ALMEIDA, 1998; SILVEIRA; SILVEIRA; DALLA-BONA, 2000).

Apesar de verificarem a reprodutibilidade intraexaminador do traçado cefalométrico, Cunha, Castilho e Médice (2002) constataram grande variação na reprodução dos fatores 1/.NA e FMA. Ainda, Silveira e Silveira (2006) avaliaram a reprodutibilidade de medidas cefalométricas realizadas por três diferentes clínicas de radiologia odontológica da cidade de Porto Alegre - RS. Os resultados obtidos de 40 telerradiografias apresentaram disparidade significativa de valores na grande maioria das medidas cefalométricas.

Conforme o apresentado na literatura, verifica-se que a análise cefalométrica não é uma ferramenta precisa, pois diferenças importantes estão presentes nas avaliações intra e interexaminadores 
(SILVEIRA; SILVEIRA; DALLA-BONA, 2000; CUNHA; CASTILHO; MÉDICI, 2002; ONGKOSUWITO et al., 2002; SILVEIRA; SILVEIRA, 2006). Frente a isso, o objetivo deste estudo foi comparar as medidas obtidas em telerradiografias sem e, posteriormente, com identificadores metálicos nos pontos cefalométricos, realizadas por um examinador treinado e calibrado.

\section{Materiais e Métodos}

Foram utilizados 10 crânios secos para a obtenção de 20 telerradiografias, sendo: 10 sem e $10 \mathrm{com}$ identificadores metálicos nos pontos anatômicos de interesse. Na Tabela 1 estão descritos os pontos anatômicos e fatores cefalométricos utilizados neste estudo. Para a realização das telerradiografias com os identificadores metálicos, usou-se esferas de aço com $1 \mathrm{~mm}$ de diâmetro fixadas com cera nos pontos anatômicos determinados, sendo que aquelas medidas cefalométricas originadas da marcação sobre os pontos metálicos foram consideradas o padrão ouro.

Tabela 1. Pontos e fatores cefalométricos utilizados no estudo.

\begin{tabular}{ll|ll}
\hline Pontos cefalométricos & Fatores cefalométricos \\
\hline $\mathrm{S}$ & Sela & SNA & \multicolumn{2}{|c}{$\begin{array}{l}\text { Posição da maxila em } \\
\text { relação à base do } \\
\text { crânio }\end{array}$} \\
\hline $\mathrm{N}$ & Násio & SNB & $\begin{array}{l}\text { Posição da mandíbula } \\
\text { em relação à base do } \\
\text { crânio }\end{array}$ \\
\hline A & Ponto A & ANB & $\begin{array}{l}\text { Posição da maxila em } \\
\text { relação à mandíbula }\end{array}$ \\
\hline B & Ponto B & FNP & $\begin{array}{l}\text { Posição da mandíbula } \\
\text { em relação ao plano } \\
\text { de Frankfurt }\end{array}$ \\
\hline Po & Pório & NAP & \begin{tabular}{l} 
Perfil do paciente \\
\hline Or
\end{tabular} \\
\hline Pog & Orbital & SNGn & $\begin{array}{l}\text { Tendência } \\
\text { crescimento }\end{array}$ \\
\hline Me & Pogônio & SNGoMe & $\begin{array}{l}\text { Padrão do esqueleto } \\
\text { cefálico }\end{array}$ \\
\hline Gn & Mentoiano & PogNB & Protrusão mentoniana \\
\hline
\end{tabular}

As mandíbulas foram fixadas aos crânios para evitar a perda da relação e promover a reprodução do posicionamento. As radiografias foram realizadas em aparelho de raios $X$ panorâmico (Rotograph Plus, Villa Sistemi Medicali, Washington, EUA, 65 kVp, 10 $\mathrm{mA}, 0,4 \mathrm{~s})$ e processados em máquina automática. Foi realizada a digitalização das radiografias por meio de um scanner de mesa com leitor de transparência. Para a identificação dos pontos e realização da análise cefalométrica foi utilizado o Sistema Radiocef®) (Radio Memory Ltda., Belo Horizonte, Minas Gerais, Brasil).

Um examinador, especialista em radiologia odontológica, foi previamente treinado quanto à definição e marcação dos pontos. A calibragem consistiu na realização de análises cefalométricas em 30 telerradiografias laterais de pacientes. A reprodutibilidade foi avaliada por meio do coeficiente de correlação intraclasse (Tabela 2).

Posteriormente à calibragem, o examinador executou as análises cefalométricas nas 20 telerradiografias do estudo, iniciando por aquelas sem os identificadores metálicos. As mensurações dos fatores cefalométricos, obtidos nos dois grupos foram comparadas entre si por meio do teste $t$ pareado $(p \leq 0,05)$ e coeficiente de repetibilidade (Bland\&Altman) (Tabela 3 ).

\section{Resultados}

Os valores do coeficiente de correlação intraclasse durante a calibragem demonstraram que o profissional apresentou boa reprodutibilidade (Tabela 2 ).

Tabela 2: Valores do coeficiente de correlação intraclasse para os fatores utilizados na calibragem do estudo.

Coeficiente de Correlação
Intraclasse

\begin{tabular}{ll}
\hline (N-Pog).(Po-Orb) & 0,88 \\
\hline N-A.Pog & 0,89 \\
\hline S-N.A & 0,70 \\
\hline S-N.B & 0,74 \\
\hline A-N.B & 0,87 \\
\hline S-N.Gn & 0,80 \\
\hline (S-N).(Go-Me) & 0,88 \\
\hline Pog-NB & 0,94 \\
\hline FMA & 0,90 \\
\hline
\end{tabular}

Os valores dos fatores cefalométricos (N-Pog).(Po-Orb), NA.P, S-N.A, S-N.B, A.N.B, S-N.Gn, (S-N).(Go-Me), Pog-NB e FMA dos grupos com e sem identificadores, quando avaliados pelo teste $t$ pareado, não apresentaram diferenças significantes. Os fatores cefalométricos que apresentaram melhor e pior concordância foram FMA e N-A.Pog, respectivamente. Já pela análise de Bland\&Altman, os fatores que apresentaram melhor e pior coeficiente de repetibilidade foram S-N.A e (S-N).(Go-Me), respectivamente (Tabela 3).

\section{Discussão}

A cefalometria é uma ferramenta importante na área da ortodontia, ortopedia e cirurgia, pois possibilita a realização de medidas de ângulos e distâncias craniofaciais antes, durante e após a conclusão do tratamento, permitindo assim, a avaliação do desenvolvimento do procedimento executado. No entanto, diversos autores discutem a falta de reprodutibilidade na identificação dos pontos anatômicos realizada nas telerradiografias, tanto quando executadas pelo mesmo quanto por diferentes profissionais. Desta forma, reitera-se a importância da educação continuada para a diminuição de erros na marcação dos pontos cefalométricos (SILVEIRA et al., 2009; DELAMARE et al., 2010).

Em relação ao tipo de imagem utilizada para a realização da cefalometria, a literatura atual indica que a tomografia computadorizada de feixe cônico, apesar das vantagens da visualização em três dimensões, sem sobreposição de estruturas, 
não apresenta superioridade de diagnóstico quando comparada à telerradiografia lateral convencional (LIEDKE et al., 2012).

Tabela 3: Médias das diferenças, valores do teste $t$ pareado, coeficiente de repetibilidade e intervalo de concordância para os fatores utilizados no estudo.

\begin{tabular}{|c|c|c|c|c|}
\hline & \multirow[b]{2}{*}{$\begin{array}{c}\text { Média } \\
\text { das } \\
\text { diferenças }\end{array}$} & \multirow{2}{*}{$\begin{array}{c}\text { Teste } t \\
\text { pareado } \\
(p \leq 0,05)\end{array}$} & \multicolumn{2}{|c|}{ Bland\&Altman } \\
\hline & & & $\begin{array}{c}\text { Coeficiente } \\
\text { de } \\
\text { Repetibilidade }\end{array}$ & $\begin{array}{l}\text { Intervalo de } \\
\text { Concordância }\end{array}$ \\
\hline $\begin{array}{l}(\mathrm{N}- \\
\text { Pog).(Po- } \\
\text { Orb) }\end{array}$ & $-0,576$ & 0,84 & 2,11 & $(-2,24 / 0,6)$ \\
\hline N-A.Pog & $-0,385$ & 0,96 & 2,82 & $(-2,48 / 2,03)$ \\
\hline S-N.A & $-0,502$ & 0,81 & 1,58 & $(-1,43 / 0,71)$ \\
\hline S-N.B & $-0,493$ & 0,82 & 1,72 & $(-1,54 / 0,76)$ \\
\hline A-N.B & $-0,58$ & 0,85 & 3,39 & $(-4,65 / 0,52)$ \\
\hline S-N.Gn & 0,43 & 0,79 & 1,72 & $(-0,87 / 1,96)$ \\
\hline $\begin{array}{l}\mathrm{S}- \\
\mathrm{N}) \cdot(\mathrm{Go}- \\
\mathrm{Me})\end{array}$ & 0,834 & 0,73 & 3,52 & $(-1,52 / 3,48)$ \\
\hline Pog-NB & 1,124 & 0,81 & 3,26 & $(-1,29 / 3,35)$ \\
\hline FMA & 0,893 & 0,7 & 3,25 & $(-0,88 / 3,7)$ \\
\hline
\end{tabular}

De acordo com Major et al. (1994) e Lau, Cooke e Hägg (1997), o erro intra-examinador é menos perceptível que o interexaminador. Assim, poder-se-ia acreditar que o problema de reprodutibilidade na identificação dos pontos anatômicos ocorre em razão da percepção de cada profissional, ou mesmo da área ou escola em que este foi treinado para a realização dos traçados cefalométricos. A solução proposta seria, de acordo com Albuquerque Júnior e Almeida (1998), a realização de calibragem prévia à execução de cefalometrias. No entanto, a realização de uma adequada calibragem pode não corresponder a uma correta identificação de pontos anatômicos, conforme verificado neste estudo.

A comparação dos valores das medidas cefalométricas com o padrão-ouro, realizadas por um examinador treinado e calibrado, demonstra que esta calibragem, previamente aceita como adequada, pode corresponder a uma reprodução de identificações equivocadas dos pontos anatômicos. Alguns estudos sugerem que a reprodução de erros do examinador ocorre em razão da concepção individual na definição e percepção dos pontos anatômicos, conforme é sugerido por Stabrun e Danielsen (1982); Lau, Cooke e Hägg (1997); Silveira, Silveira e Dalla-Bona (2000).

\section{Conclusão}

A partir dos resultados obtidos, conclui-se que, mesmo treinado e calibrado para a identificação de pontos anatômicos em telerradiografias laterais, o profissional pode gerar valores incorretos para os fatores cefalométricos. A calibragem intra-examinador executada em radiografias de pacientes, pode não refletir a certeza do acerto, e sim a repetição de erros não identificados.

\section{Referências}

ALBUQUERQUE JUNIOR, H.R.; ALMEIDA, M.H.C. Avaliação do erro de reprodutibilidade dos valores cefalométricos aplicados na filosofia Tweed-Merrifield, pelos métodos computadorizado e convencional. Ortodontia, São Paulo, v. 31, n. 3, p. 18-30, set./dez. 1998.

CUNHA, T. C. R.; CASTILHO, J. C. M.; MÉDICI FILHO, E. Avaliação da confiabilidade nas mensurações cefalométricas computadorizadas, através da reprodutibilidade do traçado cefalométrico em diferentes momentos. J. Bras. Ortodon. Ortop. Facial, Curitiba, v. 7, n. 40, p. 333-338, jul./ago. 2002.

DELAMARE E. L. et al. Influence of a programme of professional calibration in the variability of landmark identification using cone beam computed tomography-synthesized and conventional radiographic cephalograms. Dentomaxillofac. Radiol., Tokyo, v. 39, no. 7, p. 414423, Oct. 2010.

LAU, P. Y. W.; COOKE, M. S.; HÄGG, U. Effect of training and experience on cephalometric measurement errors on surgical patients. Int. J. Adult Orthodon. Orthognath. Surg., Chicago, v. 12, no. 3, p. 204-213, 1997.

LIEDKE G. S. et al. Comparative study between conventional and cone beam CT-synthesized half and total skull cephalograms. Dentomaxillofac. Radiol., Tokyo, v. 41, no. 2, p. 136-142, Feb. 2012.

MAJOR P. W. et al. Landmark identification error in posterior anterior cephalometrics. The Angle Orthodontist., Edmonton, v. 64, n. 6, p. 447-454, Dec. 1994

ONGKOSUWITO E. M. et al. The reproducibility of cephalometric measurements: a comparison of analogue and digital methods. Eur. J. Orthod., Oxford, v. 24, no. 6, p. 655-665, Dec. 2002.

RUDOLPH, D. J.; SINCLAIR, P. M.; COGGINS, J. M. Automatic computerized radiographic identification of cephalometric landmarks. Am. J. Orthod. Dentofacial Orthop., St. Louis, v. 113, no. 2, p. 173179, Feb. 1998.

SILVEIRA, H.L.D.; SILVEIRA, H.E.D. Reproducibility of cephalometric measurements made by three radiology clinics. Angle Orthod., Appleton, v. 76, no. 3, p. 394-399, May 2006.

SILVEIRA, H.L.D.; SILVEIRA, H.E.D.; DALLA-BONA, R.R. A influencia da identificação de pontos anatômicos nos resultados obtidos em análise cefalométrica. R. Fac. Odontol. Porto Alegre, Porto Alegre, v. 42, n. 2, p. 41-43, dez. 2000.

SILVEIRA, H.L.; GOMES M. J.; SILVEIRA H. E. et al. Evaluation of the radiographic cephalometry learning process by a learning virtual object. Am. J. Orthod. Dentofacial Orthop., St. Louis, v.136, no.1, p.134-138, July 2009.

STABRUN, A.E.; DANIELSEN, K. Precision in cephalometric identification. Eur. J. Orthod., Oxford, v. 4, no. 3, p. 185-196, Aug. 1982.

STEINER, C. Cephalometrics for you and me. Am. J. Orthod., St. Louis, v. 39, no. 10, p. 720-755, Oct. 1953.

YEN, P. K. J. Identification of landmarks in cephalometric radiography. Angle Orthod., Appleton, v. 30, no. 1, p. 35-41, Jan. 1960. 